\title{
Ramsey Properties of Permutations
}

\author{
Julia Böttcher* \\ Departamento de Ciência da Computação \\ Instituto de Matemática e Estatística \\ Universidade de São Paulo \\ Rua do Matão 1010 \\ 05508-090 São Paulo, Brazil
}

\author{
Jan Foniok ${ }^{\dagger}$ \\ Laboratoire d'Informatique (LIX) \\ CNRS UMR 7161 \\ Ecole Polytechnique \\ Route de Saclay \\ 91128 Palaiseau, France
}

Submitted: Mar 29th, 2011; Accepted: Dec 21, 2012; Published: Jan 7, 2013

Mathematics Subject Classifications: 05D10, 05C55, 03C52

\begin{abstract}
The age of each countable homogeneous permutation forms a Ramsey class. Thus, there are five countably infinite Ramsey classes of permutations.
\end{abstract}

Keywords: Ramsey class, permutation, homogeneous structure

\section{Introduction}

The finite Ramsey theorem [13] states that for any natural numbers $a, b$ and $r$ there is a natural number $c$ such that in any $r$-colouring of all $a$-element subsets of the set $\{1, \ldots, c\}$ there is a monochromatic $b$-element subset. This property is usually denoted by the ErdösRado partition arrow: $c \rightarrow(b)_{r}^{a}$. In the following, the least such number $c$ will be denoted by $R(a, b, r)$.

Different variants and generalisations of this result (cf. Nešetřil [12]) have been investigated. The concept of Ramsey classes is perhaps the most general of these. A Ramsey class is a class $\mathscr{K}$ of objects such that for each natural number $r$ and each choice of objects $A, B \in \mathscr{K}$ there is an object $C \in \mathscr{K}$ such that in any $r$-colouring of all subobjects of $C$ isomorphic to $A$, there is a monochromatic subobject isomorphic to $B$ :

$$
C \rightarrow(B)_{r}^{A} .
$$

We consider classes of finite relational structures, with embeddings as subobjects (for definitions see Section 2). In this case, there is a strong connection between Ramsey classes and homogeneous structures.

\footnotetext{
${ }^{*}$ Current address: Department of Mathematics, Columbia House, London School of Economics, Houghton Street, London WC2A 2AE, UK. j . boettcher@lse . ac . uk

${ }^{\dagger}$ Current address: Department of Mathematics and Statistics, Queen's University, Jeffery Hall, 48 University Avenue, Kingston ON K7L 3N6, Canada. foniok@mast . queensu. ca
} 
Theorem 1.1 (cf. Nešetřil [8]). If $\mathscr{K}$ is a hereditary isomorphism-closed Ramsey class with the joint embedding property, then $\mathbb{K}$ is the age of a countable homogeneous structure.

For investigating Ramsey classes it therefore suffices to consider classes of relational structures that are the age of some homogeneous structure. A classification programme of Ramsey classes was launched by Nešetřil [8], see also [6]. Ramsey classes of graphs had been characterised by Nešetřil [7], and characterisations for tournaments and posets appear in [8], and for posets again in $[15,16]$.

In this paper, we make a small contribution to this classification programme and determine all Ramsey classes of finite permutations. We establish the following result.

Theorem 1.2. The age of each countable homogeneous permutation forms a Ramsey class.

For the proof, we use a characterisation of homogeneous permutations by Cameron [2] (see Theorem 3.1). For the respective ages, we apply the finite Ramsey theorem, the product Ramsey theorem, and the partite construction and amalgamation technique of Nešetřil and Rödl $[10,11]$ in order to establish Theorem 1.2 (see Sections 4 and 5).

\section{Background: Homogeneous structures and Ramsey classes}

According to Theorem 1.1 homogeneous relational structures and Ramsey classes are closely connected. In this section we provide the necessary formal definitions.

We consider relational structures with a fixed signature $\sigma$. The domain of a structure $A$ is denoted by $\underline{A}$. An injective mapping $f: \underline{A} \rightarrow \underline{B}$ is an embedding of $A$ into $B$ if for every $k$-ary relation symbol $R$ in $\sigma$ and any $k$-tuple $\left(x_{j}: j=1, \ldots, k\right)$ we have $\left(x_{j}: j=1, \ldots, k\right) \in R^{A}$ iff $\left(f\left(x_{j}\right): j=1, \ldots, k\right) \in R^{B} . A$ is a substructure of $B$ if the inclusion mapping is an embedding of $A$ into $B$; we write $A \subseteq B$. Moreover, $\left(\begin{array}{c}B \\ A\end{array}\right)$ stands for the set of all embeddings of $A$ into $B$.

Consider a class $\mathscr{K}$ of relational structures that is closed under isomorphisms. For structures $A, B, C \in \mathscr{K}$ and $r \in \mathbb{N}$ the Erdős-Rado partition arrow $C \rightarrow(B)_{r}^{A}$ denotes the following: For each partition $\left(\begin{array}{l}C \\ A\end{array}\right)=\mathscr{A}_{1} \cup \cdots \cup \mathscr{A}_{r}$ there exist $i \in\{1, \ldots, r\}$ and $B^{\prime} \subseteq C$ such that $B^{\prime} \cong B$ and $\left(\begin{array}{l}B^{\prime} \\ A\end{array}\right) \subseteq \mathscr{A}_{i}$. We also call $B^{\prime}$ a monochromatic copy of $B$ in $C$ and the partition of $\left(\begin{array}{l}C \\ A\end{array}\right)$ a colouring. For $A \in \mathscr{K}$ we say that $\mathscr{K}$ is $A$-Ramsey if for every $B \in \mathscr{K}$ and $r \in \mathbb{N}$ there exists a $C \in \mathscr{K}$ such that $C \rightarrow(B)_{r}^{A}$. $\mathscr{K}$ is a Ramsey class if it is $A$-Ramsey for every $A \in \mathscr{K}$.

A relational structure $B$ is called homogeneous if every isomorphism $f: A \rightarrow A^{\prime}$ between finite substructures $A$ and $A^{\prime}$ of $B$ can be extended to an automorphism of $B$. For a structure $B$, the age of $B$ is the class of all finite structures that can be embedded into $B$.

Let $\mathscr{K}$ be a class of relational structures. Then $\mathscr{K}$ is hereditary if $A^{\prime} \in \mathscr{K}$ and $A \subseteq A^{\prime}$ imply $A \in \mathscr{K}$. We say that $\mathscr{K}$ has the joint embedding property if for any $B, B^{\prime} \in \mathscr{K}$ there is $C \in \mathscr{K}$ such that both $B$ and $B^{\prime}$ are embeddable in $C$. We say that $\mathscr{K}$ has the amalgamation property if for every $A, B, B^{\prime} \in \mathscr{K}$ and every embedding $f: A \rightarrow B$ of $A$ into $B$ and every embedding $f^{\prime}: A \rightarrow B^{\prime}$ of $A$ into $B^{\prime}$ there exists $C \in \mathscr{K}$ and embeddings $g: B \rightarrow C$ and $g^{\prime}: B^{\prime} \rightarrow C$ such that $g \circ f=g^{\prime} \circ f^{\prime}$. If the intersection of $g(\underline{B})$ and $g^{\prime}\left(\underline{B^{\prime}}\right)$ is equal to $g \circ f(\underline{A})$, we say that the amalgamation (or the joint embedding) is strong. 
Because of Theorem 1.1 we are interested in ages of countable homogeneous relational structures. A classical result of Fraïssé gives a necessary and sufficient condition for a class of finite structures to be the age of such a structure.

Theorem 2.1 (Fraïssé [4]). A class $\mathscr{K}$ of finite relational structures is the age of a countable homogeneous relational structure $\mathscr{U}$ iff the following five conditions hold:

1. $\mathscr{K}$ is closed under isomorphism,

2. $\pi$ has only countably many isomorphism classes,

3. $\mathscr{K}$ is hereditary,

4. $\dddot{K}$ has the joint embedding property,

5. $\mathscr{K}$ has the amalgamation property.

For a class $\mathscr{K}$ that satisfies the conditions 1.-5. of Theorem 2.1, the countable homogeneous structure $\mathscr{U}$ is unique up to isomorphism and is called the Fraïssé limit of $\mathscr{K}$.

\section{Permutations}

From now on we will be concerned with the proof of Theorem 1.2. We first need to introduce some notation. We adopt the definition of permutation following Cameron [2]: as a relational structure with two linear (total) orders. This definition applies to both finite and infinite permutations. Its advantage over viewing a permutation as a bijective mapping of a set to itself is that it makes the notion of subpermutation clearer.

Formally, a permutation $P=\left(X,<_{0},<_{1}\right)$ consists of a set $X$ and two linear orders $<_{0}$ and $<_{1}$ of $X$ where $<_{0}$ is called the natural order on $X$ and $<_{1}$ is arbitrary. An example is shown in Figure 1. A subpermutation of $P$ is a permutation $P^{\prime}=\left(X^{\prime},<_{0}^{\prime},<_{1}^{\prime}\right)$ with $X^{\prime} \subseteq X$ such that $<_{i}^{\prime}$ is the restriction of $<_{i}$ to $X^{\prime}$. Let $P=\left(X,<_{0},<_{1}\right)$ be a finite permutation with $|X|=x$ and consider a sequence $\left(p_{i}: i=1, \ldots, x\right)$ of pairwise distinct numbers $p_{i} \in\{1, \ldots, x\}$. Then we call $\left(p_{i}: i=1, \ldots, x\right)$ the pattern of $P$ if $P$ is isomorphic to the permutation $P^{\prime}=\left(\{1, \ldots, x\},<,<_{1}^{\prime}\right)$ with $i<j$ iff $p_{i}<_{1}^{\prime} p_{j}$ for $i, j \in\{1, \ldots, x\}$.

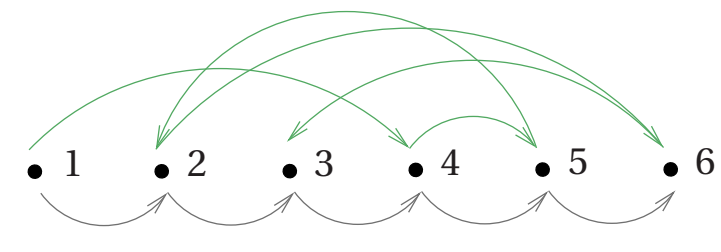

Figure 1: The permutation with pattern 1,4, 5, 2, 6, 3 (transitive arcs are omitted).

The countable homogeneous permutations were characterised by Cameron [2]. Let $\mathbf{1}$ be the unique permutation on one point. An identity permutation is a permutation of the form $\left(X,<_{0},<_{0}\right)$ and a reversal is a permutation of the form $\left(X,<_{0},>_{0}\right)$. An increasing sequence of decreasing sequences is a permutation that contains no subpermutation with pattern $2,3,1$ and no subpermutation with pattern 3,1,2. Decreasing sequences of increasing sequences are defined analogously (no 2, 1,3 and no 1,3,2). 
Theorem 3.1 (Cameron [2]). The age of each countable homogeneous permutation is one of the following classes of finite permutations:

1. $\{\mathbf{1}\}$,

2. the class of identity permutations,

3. the class of reversals,

4. increasing sequences of decreasing sequences,

5. decreasing sequences of increasing sequences,

6. the class of all permutations.

The corresponding countable homogeneous permutations (Fraïssé limits), described by Cameron [2] as well, are the following:

1. 1,

2. $(\mathbb{Q},<,<)$,

3. $(\mathbb{Q},<,>)$,

4. $\left(\mathbb{Q}^{2},<_{0},<_{1}\right)$, where $<_{0}$ is the lexicographic ordering and $<_{1}$ is the lexicographic ordering reversed within blocks,

5. $\left(\mathbb{Q}^{2},<_{0},<_{1}\right)$, where $<_{0}$ is the lexicographic ordering and $<_{1}$ is the lexicographic ordering reversed between blocks,

6. $\left(\mathbb{Q}^{2},<_{0},<_{1}\right)$, where $(x, y)<_{0}(u, v)$ if $x a+y b<u a+v b$, and $(x, y)<_{1}(u, v)$ if $x c+y d<$ $u c+v d ; a, b, c, d$ are fixed real numbers such that $b / a$ and $d / c$ are distinct irrationals satisfying $b / a+d / c \neq 0$.

Our main theorem, Theorem 1.2, claims that each of the classes of Theorem 3.1 forms a Ramsey class (and hence, because of Theorem 1.1, these are the only Ramsey classes of permutations). As a direct consequence of the finite Ramsey theorem we have the following.

Fact. The class of identity permutations and the class of reversals are Ramsey classes.

In the rest of this paper we thus study the remaining three classes from Theorem 3.1.

\section{Increasing and decreasing sequences and the product Ramsey theorem}

First, we investigate the class of increasing sequences of decreasing sequences and the class of decreasing sequences of increasing sequences. For showing that they are Ramsey we make use of the product Ramsey theorem of Graham, Rothschild and Spencer [5, Chapter 5.1]. Let $X$ be a set. We write $\left(\begin{array}{l}X \\ k\end{array}\right)$ for the set of all $k$-element subsets of $X$.

Theorem 4.1 (product Ramsey theorem). For all $r, t, n \in \mathbb{N}$ and $p \in \mathbb{N}^{t}$ there exists $N \in \mathbb{N}$ such that the following holds: If

- $X_{1}, X_{2}, \ldots, X_{t}$ are sets such that $\left|X_{i}\right| \geqslant N$ for all $1 \leqslant i \leqslant t$, and

- we r-colour $\left(\begin{array}{l}X_{1} \\ p_{1}\end{array}\right) \times \cdots \times\left(\begin{array}{l}X_{t} \\ p_{t}\end{array}\right)$,

then there are $Y_{i} \subseteq X_{i}$ with $\left|Y_{i}\right| \geqslant n$ for all $1 \leqslant i \leqslant t$ such that $\left(\begin{array}{l}Y_{1} \\ p_{1}\end{array}\right) \times \cdots \times\left(\begin{array}{l}Y_{t} \\ p_{t}\end{array}\right)$ is monochromatic. 
In the following let $P R(r, p, t, n)$ denote the least integer $N$ such that the assertion of this theorem holds for 'input' $r, p, t, n$.

Next, we use the product Ramsey theorem in order to deduce the following variant for sets $A \subseteq \mathbb{N}^{2}$ of ordered pairs, which applies more directly to our problem. Two finite sets $A \subseteq \mathbb{N} \times J$ and $A^{\prime} \subseteq \mathbb{N} \times J^{\prime}$ with $J=\left\{j_{1}, \ldots, j_{n}\right\} \subseteq \mathbb{N}$ and $J^{\prime}=\left\{j_{1}^{\prime}, \ldots, j_{n^{\prime}}^{\prime}\right\} \subseteq \mathbb{N}$ where $j_{1}<\cdots<j_{n}$ and $j_{1}^{\prime}<\cdots<j_{n^{\prime}}^{\prime}$ are isomorphic if $n=n^{\prime}$ and $\left|A \cap\left(\mathbb{N} \times\left\{j_{i}\right\}\right)\right|=\left|A^{\prime} \cap\left(\mathbb{N} \times\left\{j_{i}^{\prime}\right\}\right)\right|$ for all $1 \leqslant i \leqslant n$. Moreover, $A^{\prime}$ is a substructure of $B$ if it is a subset of $B$, and $\left(\begin{array}{l}B \\ A\end{array}\right)$ contains all substructures $A^{\prime}$ of $B$ that are isomorphic to $A$.

Theorem 4.2. For all $r, t(A), t(B) \in \mathbb{N}$ and all $a \in \mathbb{N}^{t(A)}, b \in \mathbb{N}$ there exist $t(C), c \in \mathbb{N}$ such that the following holds: If

$$
\begin{aligned}
& A=\left\{(x, j): x \in\left\{1, \ldots, a_{j}\right\}, j \in\{1, \ldots, t(A)\}\right\}, \\
& B=\{(x, j): x \in\{1, \ldots, b\}, j \in\{1, \ldots, t(B)\}\}, \\
& C=\{(x, j): x \in\{1, \ldots, c\}, j \in\{1, \ldots, t(C)\}\}
\end{aligned}
$$

and we r-colour $\left(\begin{array}{l}C \\ A\end{array}\right)$, then there is a monochromatic copy of B in $C$.

Observe that, crucially, while the sets $B$ and $C$ in this theorem can be written as the Cartesian product of two sets of integers, the first coordinate of pairs from $A$ may assume different ranges depending on the second coordinate. We will need this property for our result concerning permutations.

Proof. Set $t(C):=R(t(A), t(B), r)$ and $\gamma:=\left(\begin{array}{l}t(C) \\ t(A)\end{array}\right)$. Moreover, define $c(1):=P R(r, a, t(A), b)$ and $c(i+1):=P R(r, a, t(A), c(i))$ for $i>1$ and let $c:=c(\gamma)$. Let

$$
\begin{array}{ll}
A=A_{1} \cup \cdots \cup A_{t(A)} & \text { with } A_{i}=\left\{(x, i): x \in\left\{1, \ldots, a_{i}\right\}\right\} \text { for } 1 \leqslant i \leqslant t(A), \\
B=B_{1} \cup \cdots \cup B_{t(B)} & \text { with } B_{i}=\{(x, i): x \in\{1, \ldots, b\}\} \text { for } 1 \leqslant i \leqslant t(B), \\
C=C_{1} \cup \cdots \cup C_{t(C)} & \text { with } C_{i}=\{(x, i): x \in\{1, \ldots, c\}\} \text { for } 1 \leqslant i \leqslant t(C),
\end{array}
$$

and consider a colouring $\chi$ of $\left(\begin{array}{l}C \\ A\end{array}\right)$.

We establish the theorem by finding sets $\tilde{C}_{j} \subseteq C_{j}$ of cardinality $b$ for $1 \leqslant j \leqslant t(C)$ such that $\tilde{C}=\tilde{C}_{1} \cup \cdots \cup \tilde{C}_{j}$ has the following property: For all distinct $i_{1}, \ldots, i_{t(A)} \in\{1, \ldots, t(C)\}$ all copies of $A$ in $\tilde{C}_{i_{1}} \cup \cdots \cup \tilde{C}_{i_{t(A)}}$ have the same colour. This uniquely determines an $r$-colouring of the set $\mathscr{S}$ of all $t(A)$-element subsets of the integers $1, \ldots, t(C)$. By the choice of $t(C)$ and the finite Ramsey theorem we find a monochromatic $t(B)$-element subset in $\mathscr{S}$ and accordingly a monochromatic copy of $B$ in $C$.

To find the sets $\tilde{C}_{j}$ we repeatedly apply the product Ramsey theorem. The idea is to do the following step for each choice of distinct $i_{1}, \ldots, i_{t(A)} \in\{1, \ldots, t(C)\}$. Consider $C^{*}:=C_{i_{1}} \cup$ $\cdots \cup C_{i_{t(A)}}$ and choose the largest monochromatic subset (with respect to $\chi$ ) of $C^{*}$. For the next step, restrict each set $C_{i_{j}}$ to $C_{i_{j}} \cap C^{*}$. Then continue with the next choice of $i_{1}, \ldots, i_{t(A)}$. By the choice of $c$ and the product Ramsey theorem we will get subsets $\tilde{C}_{j}$ of $C_{j}$ with $\left|\tilde{C}_{j}\right| \geqslant b$ at the end of this procedure.

This immediately implies the desired result. 
Corollary 4.3. The class of increasing sequences of decreasing sequences and the class of decreasing sequences of increasing sequences are Ramsey classes.

Proof. Let $A, B$ be two increasing sequences of decreasing sequences. Set $t(A)$ to be the number of decreasing sequences in $A$ and let $a_{j}$ be the length of the $j$ th decreasing sequence. Furthermore set $t(B)$ to be the number of decreasing sequences in $B$ and let $b$ be the maximum length of a decreasing sequence in $B$. Apply Theorem 4.2 and let $C$ be the increasing sequence of $t(C)$ decreasing sequences of length $c$. The proof for decreasing sequences of increasing sequences is analogous.

\section{The class of all permutations}

To show that the class of all permutations is a Ramsey class we use the amalgamation technique, which was introduced by Nešetřil and Rödl $[10,11]$ (see also Nešetřil [12]). This technique consists of two main parts: the partite lemma and the partite construction. We start with some definitions.

Let $a \in \mathbb{N}$. An $a$-partite permutation $P=\left(X_{1} \cup \cdots \cup X_{a},<_{0},<_{1}\right)$ is a permutation on the union of disjoint sets $X_{1}, \ldots, X_{a}$ such that $x<_{0} y$ for $x \in X_{i}$ and $y \in X_{j}$ whenever $i<j$, and $x<_{0} y$ iff $x<_{1} y$ for $x, y \in X_{i}$ (an example is provided in Figure 2). The sets $X_{i}$ are called the parts of $P$. An $a$-partite permutation is transversal if each part is of size one. As subpermutations of $P$ we now consider only $a$-partite subpermutations $P^{\prime}=\left(X_{1}^{\prime} \cup \cdots \cup X_{a}^{\prime},<_{0}^{\prime},<_{1}^{\prime}\right)$ (where the $X_{i}^{\prime}$ are possibly empty). The trace of an $a$-partite permutation $P=\left(X_{1} \cup \cdots \cup X_{a},<_{0},<_{1}\right)$ is given by $\left\{i: X_{i} \neq \varnothing\right\}$.

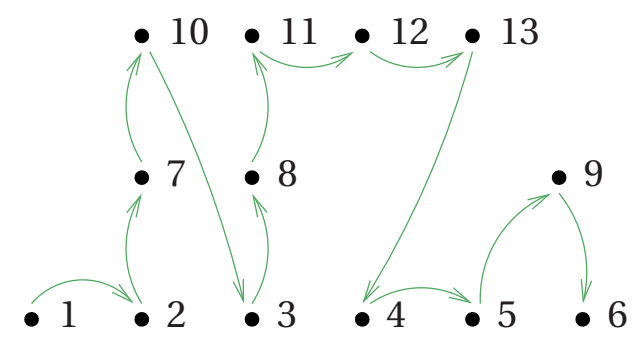

Figure 2: The 3-partite permutation with pattern 1,2,7, 10, 3, 8, 11, 12, 13, 4, 5, 9, 6 .

\subsection{The Partite Lemma}

Following the strategy of Nešetřil and Rödl we first formulate a Ramsey lemma concerning copies of $a$-partite transversals in arbitrary $a$-partite permutations. In our case this lemma asserts the following.

Lemma 5.1 (partite lemma). Let $A$ be an a-partite transversal and $B$ an arbitrary a-partite permutation. Then for any $r \in \mathbb{N}$ there exists an a-partite permutation $C$ such that $C \rightarrow(B)_{r}^{A}$. 
For the proof of this lemma we use the following class of special permutations. An $a$ snake $P=\left(X_{1} \cup \cdots \cup X_{a},<_{0},<_{1}\right)$ of length $\xi=\xi(P)$ is an $a$-partite permutation with parts

$$
X_{i}=\{(i-1) \xi+x: 1 \leqslant x \leqslant \xi\}=:\left\{1_{i}, \ldots, \xi_{i}\right\}
$$

such that $x_{i}<_{1} x_{j}$ for $0 \leqslant i<j \leqslant a$ and $1 \leqslant x \leqslant \xi$, and $x_{a}<_{1} y_{1}$ for $y=x+1$ and $1 \leqslant x<\xi$ (see Figure 3).

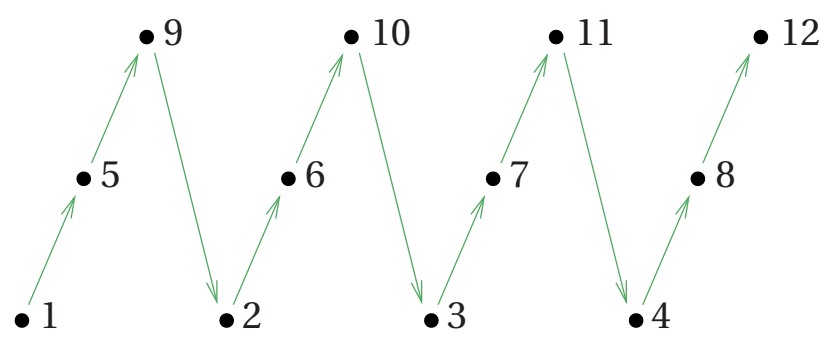

Figure 3: The 3-partite snake of length 4.

Fact. Each a-partite permutation $P=\left(X_{1} \cup \cdots \cup X_{a},<_{0},<_{1}\right)$ is a subpermutation of the a-snake of length $\sum_{i}\left|X_{i}\right|$.

For an arbitrary $a$-partite permutation $P$ let $\xi(P)$ be the length of the shortest $a$-snake that contains $P$ as a subpermutation. By the previous fact the partite lemma, Lemma 5.1, is a direct consequence of the following lemma.

Lemma 5.2. Let $A$ be an a-partite transversal and $B$ an arbitrary $a$-snake. Then for any $r \in \mathbb{N}$ there exists an a-snake $C$ such that $C \rightarrow(B)_{r}^{A}$.

Proof. We proceed by induction on $a$. The base case $a=1$ is trivial. For the induction step let $\xi\left(r, A^{\prime}, B^{\prime}\right)$ be the length of a snake $C$ such that the lemma holds for $r$ colours and $(a-1)$ partite $A^{\prime}$ and $B^{\prime}$. Now, consider an $a$-partite transversal $A$ and an $a$-snake $B$ and assume that the first element of $A$ is in part $\underline{A}_{1}$. The $(a-1)$-partite permutation induced on $\underline{A} \backslash \underline{A}_{1}$ is denoted by $A^{*}$ (and the trivial 1-partite permutation induced on $\underline{A}_{1}$ by $A_{1}$ ). Let $C$ be the $a$-partite snake of length

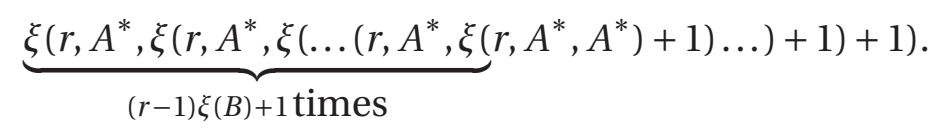

We claim that $C$ has the desired properties. Indeed, consider a colouring $\chi$ of $\left(\begin{array}{l}C \\ A\end{array}\right)$. Let $\underline{C}_{1}$ be the part of $C$ that corresponds to $\underline{A}_{1}$, let $C_{1}$ be the 1-partite permutation induced on $\underline{C}_{1}$, and $C^{*}$ the $(a-1)$-partite permutation induced on $\underline{C} \backslash \underline{C}_{1}$. Choose the first element $c_{1}$ of $C_{1}$ and consider copies of $A$ in $C$ that start in $c_{1}$. These copies of $A$ are in one to one correspondence with copies of $A^{*}$ in $C^{*}$. Let $\chi^{*}$ be the corresponding colouring of $\left(\begin{array}{c}C^{*} \\ A^{*}\end{array}\right)$, i.e., 
$\chi^{*}\left(A^{*}\right):=\chi\left(A^{*} \cup c_{1}\right)$. By the choice of $C$ and the induction hypothesis we find an $(a-1)-$ snake in $C^{*}$ that is monochromatic under $\chi^{*}$ and has length

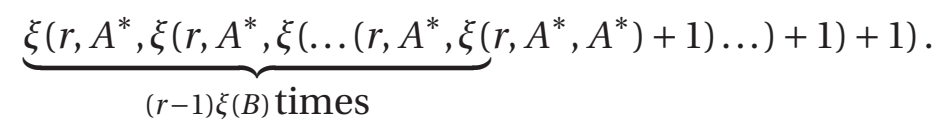

We continue by restricting $C$ to the elements of this snake and appropriate intermediate elements in $C_{1}$ and repeat this process $(r-1) \xi(B)+1$ times. Let $\tilde{C}$ be the $a$-partite snake that has in part $\underline{C}_{1}$ all the elements that were chosen as $c_{1}$ in this process. By construction, $\xi(\tilde{C})=(r-1) \xi(B)+1$. Observe, moreover, that the colour of a copy of $A$ in $\tilde{C}$ depends only on its element in $A_{1}$. By the pigeon hole principle we therefore get a monochromatic copy of $B$ in $\tilde{C}$.

\subsection{The Partite Construction}

For the partite construction we additionally need the following lemma.

Lemma 5.3. For each $a \in \mathbb{N}$ the class of a-partite permutations has the amalgamation property.

Proof. It is easy to see that the class of all permutations has strong amalgamation (recall the definition from Section 2). Hence, to amalgamate $a$-partite permutations, amalgamate the underlying permutations strongly in such a way that within one part the two orderings coincide. Membership in parts is preserved.

With this we are now ready to deduce from the partite lemma via the partite construction that the class of all permutations is Ramsey.

Theorem 5.4. The class of all permutations is a Ramsey class.

Proof. Let $r \geqslant 2$ be an integer and $A$ and $B$ be arbitrary permutations of sizes $a$ and $b$, respectively. We need to show that there is a permutation $C$ with $C \rightarrow(B)_{r}^{A}$. For this purpose let $q=R(a, b, r)$ and $\gamma=\left(\begin{array}{c}q \\ a\end{array}\right)$. In the partite construction we will recursively define permutations $P_{0}, \ldots, P_{\gamma}$ and $P_{\gamma}$ will be the permutation $C$ in quest.

We consider $A$ as an $a$-partite permutation and $B$ as a $b$-partite permutation. Let $P_{0}$ be a $q$-partite permutation such that any arbitrarily chosen $b$ parts of $P_{0}$ induce a copy of $B$. Such a permutation can easily be constructed, for instance, by concatenating $\left(\begin{array}{l}q \\ b\end{array}\right)$ copies of $B$ 'spread' over the right parts.

Let $\left(\begin{array}{c}\{1, \ldots, q\} \\ a\end{array}\right)=\left\{M_{1}, \ldots, M_{\gamma}\right\}$. For $i \geqslant 1$, each permutation $P_{i}$ is then obtained from $P_{i-1}$ by performing the following construction: Let $D_{i}$ be the permutation induced on the parts enumerated by $M_{i}$. According to the partite lemma, Lemma 5.1, there is a $b$-partite permutation $E_{i}$ such that $E_{i} \rightarrow\left(D_{i}\right)_{r}^{A}$. Construct $P_{i}$ by taking $\left|\left(\begin{array}{c}E_{i} \\ D_{i}\end{array}\right)\right|$ copies of $P_{i-1}$ and amalgamating them along the copies of $D_{i}$ in $E_{i}$. This is possible by Lemma 5.3.

Then $P_{\gamma} \rightarrow(B)_{r}^{A}$. This is shown by backward induction: Consider a colouring $\chi$ of $P_{\gamma}$ and let $P_{\gamma}^{*}=P_{\gamma}$. From $P_{i+1}^{*}$ choose a copy $P_{i}^{*}$ of $P_{i}$ such that all copies of $A$ in the corresponding 
copy of $D_{i}$ have the same colour. $P_{i}^{*}$ exists by construction (because $\left.E_{i} \rightarrow\left(D_{i}\right)_{r}^{A}\right)$. Eventually, we obtain a copy $P_{0}^{*}$ of $P_{0}$ with the following property: The colour of a copy of $A$ in $P_{0}^{*}$ depends only on its trace. Accordingly the colouring $\chi$ of $P_{0}^{*}$ induces a colouring of subsets of size $a$ of the first $q$ positive integers. By the choice of $q$ and the Ramsey theorem we therefore find a monochromatic copy of $B$ in this copy of $P_{0}$.

\section{Concluding remarks}

We have recently been informed that some of our results have independently been obtained by Sokić $[14,17]$. An alternative proof of our results, using topological properties of automorphism groups, can be obtained as an application of Bodirsky's recent manuscript [1].

The class of all structures consisting of 3 or more total orders is also a Ramsey class; this can be proved either by a modification of our proof or by the cross-construction of Sokić [14]. However, at present the classification of all homogeneous structures with $k$ total orders is open for $k \geqslant 3$.

The partite construction seems to be a promising method for proving Ramsey properties of more general amalgamation classes. The applications we have in mind include, e.g., classes of structures defined by forbidding the existence of a homomorphism from a set of finite connected structures. Such classes were studied by Cherlin, Shelah and Shi [3]. For finite sets of forbidden structures, the solution was announced by Nešetřil [9].

\section{Acknowledgements}

This research was in part done during the 2005 Prague Doccourse 'Modern Methods in Ramsey Theory', supported by the EU Research Network COMBSTRU and by DIMATIA. We would like to thank the organisers for an instructive and inspiring event, and the funding bodies for financial support. In particular we would like to thank Jarik Nešetřil for suggesting the problem to work on and for intense discussions.

The first author was financially supported by CNPq (Proc. 484154/2010-9) and by FAPESP (Proc. 2009/17831-7), and is grateful to NUMEC/USP, Núcleo de Modelagem Estocástica e Complexidade of the University of São Paulo, for supporting this research.

The second author was supported by ERC Starting Grant 'CSP-Complexity’ no. 257039 and by the Institute for Theoretical Computer Science (ITI), project 1M0545 of the Ministry of Education of the Czech Republic.

\section{References}

[1] M. Bodirsky. New Ramsey classes from old. arXiv:1204.3258 [math.LO], 2012.

[2] P. J. Cameron. Homogeneous permutations. Electron. J. Combin., 9(2):\#R2, 2002.

[3] G. Cherlin, S. Shelah, and N. Shi. Universal graphs with forbidden subgraphs and algebraic closure. Adv. in Appl. Math., 22(4):454-491, 1999. 
[4] R. Fraïssé. Theory of relations, volume 118 of Studies in logic and the foundations of mathematics. North-Holland, 1986.

[5] R. L. Graham, B. L. Rothschild, and J. H. Spencer. Ramsey Theory. J. H. Wiley, New York, second edition, 1990.

[6] J. Hubička and J. Nešetřil. Finite presentation of homogeneous graphs, posets and Ramsey classes. Israel J. Math., 149:21-44, 2005.

[7] J. Nešetřil. For graphs there are only four types of hereditary Ramsey classes. J. Combin. Theory Ser. B, 46(2):127-132, 1989.

[8] J. Nešetřil. Ramsey classes and homogeneous structures. Combin. Probab. Comput., 14(1-2):171-189, 2005.

[9] J. Nešetřil. First order definable Ramsey classes. Workshop on Homogeneous Structures, July 2011. School of Mathematics, University of Leeds.

[10] J. Nešetřil and V. Rödl. Simple proof of the existence of restricted Ramsey graphs by means of a partite construction. Combinatorica, 1(2):199-202, 1981.

[11] J. Nešetřil and V. Rödl. Two proofs of the Ramsey property of the class of finite hypergraphs. European J. Combin., 3(4):347-352, 1982.

[12] J. Nešetřill. Ramsey theory. In R. L. Graham, M. Grötschel, and L. Lovász, editors, Handbook of Combinatorics, volume 2, chapter 25, pages 1331-1403. Elsevier, 1995.

[13] F. P. Ramsey. On a problem of formal logic. Proc. London Math. Soc. (2), 30(1):264-286, 1930.

[14] M. Sokić. Ramsey property of posets and related structures. PhD thesis, University of Toronto, 2010.

[15] M. Sokić. Ramsey properties of finite posets. Order, 29(1):1-30, 2012.

[16] M. Sokić. Ramsey properties of finite posets II. Order, 29(1):31-47, 2012.

[17] M. Sokić. Ramsey property, ultrametric spaces, finite posets, and universal minimal flows. Israel J. Math., 2012, to appear. 